This item was submitted to Loughborough's Research Repository by the author.

Items in Figshare are protected by copyright, with all rights reserved, unless otherwise indicated.

\title{
Reduced-order disturbance observer design for discrete-time linear stochastic systems
}

PLEASE CITE THE PUBLISHED VERSION

http://dx.doi.org/10.1177/0142331216634425

\section{PUBLISHER}

(C) The Authors. Published by Sage.

\section{VERSION}

AM (Accepted Manuscript)

\section{PUBLISHER STATEMENT}

This work is made available according to the conditions of the Creative Commons Attribution-NonCommercialNoDerivatives 4.0 International (CC BY-NC-ND 4.0) licence. Full details of this licence are available at: https://creativecommons.org/licenses/by-nc-nd/4.0/

\section{LICENCE}

CC BY-NC-ND 4.0

\section{REPOSITORY RECORD}

Su, Jinya, Baibing Li, Wen-Hua Chen, and Jun Yang. 2016. "Reduced-order Disturbance Observer Design for Discrete-time Linear Stochastic Systems”. figshare. https://hdl.handle.net/2134/21208. 


\title{
Reduced-Order Disturbance \\ Observer Design for \\ Discrete-Time Linear \\ Stochastic Systems
}

Journal Title
$X X(X): 1-18$
C)The Author(s) 2016
Reprints and permission:
sagepub.co.uk/journalsPermissions.nav
DOI: $10.1177 / T o B e A s s i g n e d$
www.sagepub.com/

@SAGE

\section{Jinya $\mathrm{Su}^{1}$, Baibing $\mathrm{Li}^{2}$, Wen-Hua Chen ${ }^{1}$ and Jun Yang ${ }^{3}$}

\begin{abstract}
The conventional disturbance observers for discrete-time linear stochastic systems assume that the system states are fully estimable and the disturbance estimate is dependent on the estimated system states, hereafter termed Full-Order Disturbance Observers (FODOs). This paper investigates the design of Reduced-Order Disturbance Observers (RODOs) when the system state variables are not fully estimable. An existence condition of RODO is established, which is shown to be more easily satisfied than that of the conventional FODOs and consequently it has substantially extended the scope of applications of disturbance observer theory. Then a set of recursive formulae for the RODO is developed for on-line applications. Finally, it is further shown that the conventional FODOs are a special case of the proposed RODO in the sense that the former reduces to the RODO when the states become fully estimable in the presence of disturbances. Examples are given to demonstrate the effectiveness and advantages of the proposed approach.
\end{abstract}

\section{Keywords}

Disturbance Observer, Existence, Kalman filter, Reduced-order, Unknown input.

\footnotetext{
${ }^{1}$ Department of Aeronautical and Automotive Engineering, Loughborough University, Loughborough LE11 3TU, U.K.

${ }^{2}$ School of Business and Economics, Loughborough University, Loughborough LE11 3TU, U.K.

${ }^{3}$ School of Automation, Southeast University, Nanjing 210096, China.
}

\section{Corresponding author:}

Tel.: +44-1509-228841; fax: +44-1509-223960.

Email: B.Li2@lboro.ac.uk (Baibing Li) 


\section{Introduction}

In the recent years, Disturbance Observers (DOs) have received much attention in both academia and industry (see, recent books Guo and Cao (2013) and Li et al (2014), and tutorial Chen et al (2016) for recent development), and have been applied in many different areas, such as disturbance rejection control for different kinds of motion control systems including robotic manipulator, motor control (e.g., Ohishi et al (1987); Chen et al (2000); Su et al (2013); Yang et al (2013, 2014)) and fault diagnosis system design (see, Jiang and Chowdhury (2005); Su et al (2016), among many others). Based on the availability of the state information, the existing DOs can be broadly classified into three categories: (a) full measurable state based DOs (e.g., Chen et al (2000); Kim et al (2010)); (b) full state estimation based DOs (termed as FODOs) (e.g., Gillijns and De Moor (2007); Fang et al (2013); Su et al (2015b)); and (c) reduced-order state function estimation based DOs (termed as RODOs) (e.g., Xiong and Saif (2003); Kim and Rew (2013)).

In this paper, we focus on the RODO design. Practically, there are three major reasons why a RODO is needed. First, in areas such as fault diagnosis, an estimate of the entire states may not be necessary for the purpose of fault estimation (Xiong and Saif (2003)). Secondly, there are some practical scenarios where disturbance estimation is required even if the states are not fully estimable (Bejarano et al (2009)). Finally, when a fast disturbance estimate is required, DOs with a lower order are often more desirable for on-line implementation (Ohishi et al (1987)).

The conventional disturbance observers assume that all the system states are estimable or even directly measurable (e.g., Chen et al (2000); Kim et al (2010); Su et al (2015b)), and consequently the disturbance estimation is dependent on the estimated system states. For example, the researchers in Ohishi et al (1987) proposed a DO by treating the disturbances as additional states and estimating them using a deadbeat function observer (Kimura (1978)) under the assumption that the augmented systems are completely observable and the disturbances can be approximated by known transition dynamics. A proportional integral observer was used in Chang (2006) for simultaneous estimation of the system states and unknown disturbances under slow-varying disturbances and extended state observability assumptions. On the other hand, to relax the assumption on disturbances and incorporate noise information for stochastic systems, Gillijns and De Moor (2007) proposed a simultaneous state and disturbance observer on the basis of Darouach and Zasadzinski (1997) using the Minimum-VarianceUnbiased-Estimation (MVUE) method. Later, Su et al (2015b) extends the results to the case where information on the disturbances is available at an aggregate level ( $\mathrm{Li}$ (2013)). The assumption that the states are fully estimable inevitably restricts the applications of the FODOs (see, Su et al (2015a) for the existing condition of this kind of filter). An important earlier work on RODO can be traced back to Xiong and Saif (2003) where the concept of state function observer based on Lyapunov approach was 
investigated for continuous-time systems. Recently, a RODO has been proposed in Kim and Rew (2013) by combining a state function estimator of minimal order and a full measurable state based DO (Kim et al (2010)) for discrete-time deterministic system. The existence condition in Kim and Rew (2013), however, involves a static output feedback problem, for which the general solvability is not known yet. It also depends on an assumption that the disturbances are slowly time-varying, which will lead to large estimation error in the presence of unknown fast time-varying disturbances.

This paper aims to design a RODO for discrete-time linear stochastic systems without imposing any assumption on the disturbance dynamics. Compared with the existing FODOs, a simpler criterion for the existence of RODO is developed and the full state estimableness condition is removed. In addition, in comparison with the existing discrete-time RODOs, no assumption is made on disturbances and consequently it can obtain better disturbance estimation performance for generic disturbances. Hence it extends the applicability of the existing results in Gillijns and De Moor (2007); Darouach and Zasadzinski (1997); Li (2013); Su et al (2015a,b) to a much wider application area.

Let $I$ denote an identity matrix with suitable dimensions. Throughout the paper, $X^{+}, X^{T}$ and $X^{\perp}$ denote the Moore-Penrose Pseudo-inverse, transpose and an orthogonal complement of matrix $X$ respectively. $\operatorname{Rank}(X), \operatorname{Tr}(X)$ and $\lambda(X)$ denote the rank, trace and any of the eigenvalues of matrix $X$. In particular, when $X$ has a full column-rank, we have: (a) $X^{+}=\left(X^{T} X\right)^{-1} X^{T}$ such that $X^{+} X=I$; and (b) $X^{\perp}$ has a full column-rank and satisfies $X^{T} X^{\perp}=0$. Similarly, if $X$ has a full row-rank, we have $X^{+}=X^{T}\left(X X^{T}\right)^{-1}$ such that $X X^{+}=I$.

\section{Problem statement}

Consider a discrete-time linear stochastic system in the presence of disturbances (e.g., Gillijns and De Moor (2007); Darouach and Zasadzinski (1997); Li (2013)) as follows:

$$
\left\{\begin{array}{l}
x_{k+1}=A x_{k}+G d_{k}+\omega_{k} \\
y_{k}=C x_{k}+v_{k}
\end{array},\right.
$$

where $x_{k}=\left[x_{k, 1}, \ldots, x_{k, n}\right]^{T} \in \mathbb{R}^{n}$ is the state vector, $d_{k}=\left[d_{k, 1}, \ldots, d_{k, m}\right]^{T} \in \mathbb{R}^{m}$ is a vector of the lumped unknown disturbances which may include parameter uncertainties, external disturbances and system faults, and $y_{k} \in \mathbb{R}^{p}$ is the measurement vector at each time step $k$ with and $n \geq m$ and $p \geq m$. Note that the latter assumption can be relaxed when some certain prior information on the disturbances $d_{k}$ is available; interested readers may refer to $\mathrm{Su}$ et al (2015a), Chang (2006) for different types of prior disturbance information. The process noise $\omega_{k} \in \mathbb{R}^{n}$ and measurement noise $v_{k} \in \mathbb{R}^{p}$ are assumed to be mutually independent, and each follows a Gaussian distribution with a zero-mean vector and known 
covariance matrix, $Q_{k}=E\left[\omega_{k} \omega_{k}^{T}\right]>0$ and $R_{k}=E\left[v_{k} v_{k}^{T}\right]>0$ respectively. In addition, $A, G$ and $C$ are known matrices, where $G$ is supposed to have a full column-rank (see, Darouach and Zasadzinski (1997); Li (2013); Su et al (2015a) for the rationality of this assumption). The initial state $x_{0}$ is independent of $\omega_{k}$ and $v_{k}$ with a known mean $\hat{x}_{0}$ and covariance matrix $P_{0}>0$.

In general, the objective of a DO is to estimate the disturbance vector $d_{k}$ based on the measurement output $y_{k}$ and model (1). This paper, however, focuses on the design of RODO, aiming to: (a) remove the assumption of full state estimableness; (b) remove the assumption on disturbance dynamics; (c) increase the estimation speed with a lower observer order.

\section{Reduced-order disturbance observer}

In this section, we first establish an existence condition of a general RODO for system (1) when the full state vector is not estimable. This is undertaken observing the fact that one can still estimate the disturbances using the information of the estimable part of the state vector (e.g., Bejarano et al (2009); Kim and Rew (2013)). To this end, a reduced-order state function observer is used for disturbance estimation. Then on the basis of the existence condition, we derive a set of recursive formulae for the RODO.

\section{Existence condition}

Define $\mathscr{L}=\left\{l \mid A l=\lambda l\right.$ and $C l=0$, with $l \in \mathbb{R}^{n}$ and $\lambda$ is a scalar $\}$ to be a set of eigenvectors of $A$ that are orthogonal to $C^{T}$. Suppose there are in total $n_{1}$ linearly independent vectors in $\mathscr{L}$. Now, let $l_{1}, l_{2}, \cdots, l_{n_{1}}$ denote any of $n_{1}$ linearly independent vectors in $\mathscr{L}$ and let $L^{T}=\left[l_{1}, l_{2}, \cdots, l_{n_{1}}\right]$ be an $n \times n_{1}$ matrix. In addition, define $T$ to be an $\left(n-n_{1}\right) \times n$ matrix such that $T^{T}$ is an orthogonal compliment of matrix $L^{T}$ satisfying $T L^{T}=0$.

Let $z_{k}=T x_{k}$. Then the dynamics of $z_{k}$ are

$$
\begin{aligned}
z_{k+1} & =T x_{k+1}=T A x_{k}+T G d_{k}+T \omega_{k} \\
& =T A T^{+} z_{k}+T A\left(I-T^{+} T\right) x_{k}+T G d_{k}+T \omega_{k} \\
& =T A T^{+} z_{k}+T A\left(L^{+} L\right) x_{k}+T G d_{k}+T \omega_{k} .
\end{aligned}
$$

Noting that $L^{+}=L^{T}\left(L L^{T}\right)^{-1}$ and each column of matrix $L^{T}$ is an eigenvector of $A$ that is orthogonal to $T$, we have $T A L^{+} L=T A L^{T}\left(L L^{T}\right)^{-1} L=0$. Hence, we can obtain

$$
z_{k+1}=T A T^{+} z_{k}+T G d_{k}+T \omega_{k} .
$$


In addition, noting that $C L^{T}=0$, a similar argument can be applied to the measurement equation of (1), yielding

$$
y_{k}=C A T^{+} z_{k-1}+C G d_{k-1}+C \omega_{k-1}+v_{k} .
$$

For the scenario that $x_{k}$ is not fully estimable, we will estimate $d_{k}$ based on (2) and (3).

First, motivated by the linear filter structure in Gillijns and De Moor (2007), a general disturbance observer structure for system (2) and (3) is designed as

$$
\left\{\begin{array}{l}
\hat{z}_{k+1}=E_{k} \hat{z}_{k}+K_{k+1} y_{k+1} \\
\hat{d}_{k}=J_{k+1}\left(y_{k+1}-N \hat{z}_{k}\right)
\end{array},\right.
$$

where the matrices $E_{k}, K_{k+1}, J_{k+1}$ and $N$ are to be designed (and as it will be shown later, the matrix $N$ is time-invariant).

Based on (2)-(4), one can obtain the dynamics of the state function estimation error, $e_{k+1}=$ $z_{k+1}-\hat{z}_{k+1}$, as

$$
\begin{aligned}
e_{k+1}= & T A T^{+} z_{k}+T G d_{k}+T \omega_{k}-\left(E_{k} \hat{z}_{k}+K_{k+1} y_{k+1}\right) \\
= & E_{k} e_{k}+\left(T A T^{+}-K_{k+1} C A T^{+}-E_{k}\right) z_{k} \\
& +\left(T G-K_{k+1} C G\right) d_{k}+\left(T-K_{k+1} C\right) \omega_{k}-K_{k+1} v_{k+1} .
\end{aligned}
$$

The disturbance estimate is governed by

$$
\begin{aligned}
\hat{d}_{k} & =J_{k+1}\left(C A T^{+} z_{k}+C G d_{k}+C \omega_{k}+v_{k+1}-N \hat{z}_{k}\right) \\
& =J_{k+1} N e_{k}+J_{k+1}\left(C A T^{+}-N\right) z_{k}+J_{k+1} C G d_{k}+J_{k+1}\left(C \omega_{k}+v_{k+1}\right) .
\end{aligned}
$$

We first focus on Eq. (6). To ensure an unbiased disturbance estimate, $\hat{d}_{k}$ must be independent of the term $z_{k}$, and matrix $J_{k+1}$ has to satisfy $J_{k+1} C G=I$. In addition, for (5), we note that the effect of $e_{k}$ on $\hat{d}_{k}$ should disappear as $k$ increases, and hence it is required that the filtering error $e_{k}$ in (5) is independent of $z_{k}$ and $d_{k}$. Moreover, the error $e_{k}$ should also approach to zero as time $k$ increases, i.e., $E_{k}$ is a stable matrix. Therefore the existence condition for RODO (4) is summarized as follows:

(i) $E_{k}$ is stable (i.e., all the eigenvalues of $E_{k}$ satisfy $\left|\lambda\left(E_{k}\right)\right|<1$ );

(ii) $E_{k}=T A T^{+}-K_{k+1} C A T^{+}$;

(iii) $K_{k+1} C G=T G$;

(iv) $N=C A T^{+}$;

(v) $J_{k+1} C G=I$. 
For system (1) with disturbance observer (4), however, the existence condition should be expressed in terms of matrices $A, G, C$ and $T$. We provide a condition for the existence of a general linear DO (4). Theorem 1. Suppose $G$ has a full column-rank. A sufficient condition for the existence of a general RODO (4) for system (1) is that

$$
\operatorname{rank}(C G)=m
$$

and the matrix

$$
P=\left[\begin{array}{cc}
z I_{n_{1}}-T A T^{+} & -T G \\
C A T^{+} & C G
\end{array}\right]
$$

has a full column-rankfor all $z \in \mathbb{C}$ such that $|z| \geq 1$.

Proof: First, we select $E_{k}$ based on condition part (ii) as

$$
E_{k}=T A T^{+}-K_{k+1} C A T^{+}
$$

and $N=C A T^{+}$based on condition part (iv). We can further choose $J_{k+1}$ such that $J_{k+1} C G=I$ since $C G$ has a full column rank. In addition, we can obtain from Kitanidis (1987) that condition (7) guarantees there exists a matrix $K_{k+1}$ such that condition part (iii) holds. Hence, we only have to focus on condition part (i) with the constraint on $K_{k+1}$ given by condition part (iii). Since $C G$ has a full column-rank, there exists an invertible matrix $M \in R^{p \times p}$ (Su et al (2015a)) such that

$$
M C G=\left[\begin{array}{c}
0_{(p-m) \times m} \\
I_{m}
\end{array}\right] .
$$

From (iii), the general solution $K_{k+1}$ can be expressed as:

$$
K_{k+1}=\left[\Gamma_{k}, T G\right] M
$$

where $\Gamma_{k}$ can be any matrix with suitable dimension and is to be designed for the gain matrix $K_{k+1}$. Define $S_{1}$ and $S_{2}$ as

$$
\left[\begin{array}{l}
S_{1} \\
S_{2}
\end{array}\right]=M C A T^{+} .
$$

Inserting (10) into (9) gives

$$
\begin{aligned}
E_{k} & =T A T^{+}-K_{k+1} C A T^{+}=T A T^{+}-\left[\Gamma_{k}, T G\right] M C A T^{+} \\
& =T A T^{+}-\left[\Gamma_{k}, T G\right]\left[\begin{array}{c}
S_{1} \\
S_{2}
\end{array}\right]=T A T^{+}-T G S_{2}-\Gamma_{k} S_{1} .
\end{aligned}
$$


According to Anderson and Moore (2012) (see, pp. 342), existence condition part (i) holds if and only if either one of the equivalent conditions holds:

(a) $T A T^{+}-T G S_{2}-\Gamma_{k} S_{1}$ is stable for a matrix $\Gamma_{k}$;

(b) $S_{1} \eta=0$ and $\left(T A T^{+}-T G S_{2}\right) \eta=\lambda \eta$ for some constant $\lambda$ and vector $\eta$ implies $|\lambda|<1$ or $\eta=0$.

Condition (b) can be equivalently expressed as:

$$
\operatorname{rank}\left(\left[\begin{array}{c}
z I_{n_{1}}-T A T^{+}+T G S_{2} \\
S_{1}
\end{array}\right]\right)=n_{1}, \quad \forall z \in \mathbb{C},|z| \geq 1 .
$$

The following identity shows that (11) is satisfied if condition (8) holds:

$$
\begin{aligned}
& \operatorname{rank}\left(\left[\begin{array}{cc}
z I_{n_{1}}-T A T^{+} & -T G \\
C A T^{+} & C G
\end{array}\right]\right)=\operatorname{rank}\left(\left[\begin{array}{cc}
I_{n_{1}} & 0_{n_{1} \times p} \\
0_{m \times n_{1}} & M
\end{array}\right]\left[\begin{array}{cc}
z I_{n_{1}}-T A T^{+} & -T G \\
C A T^{+} & C G
\end{array}\right]\right) \\
& =\operatorname{rank}\left(\left[\begin{array}{cc}
z I_{n_{1}}-T A T^{+} & -T G \\
M C A T^{+} & M C G
\end{array}\right]\right)=\operatorname{rank}\left(\left[\begin{array}{cc}
z I_{n_{1}}-T A T^{+} & -T G \\
S_{1} & 0_{(p-m) \times m} \\
S_{2} & I_{m}
\end{array}\right]\right) \\
& =\operatorname{rank}\left(\left[\begin{array}{cc}
z I_{n_{1}}-T A T^{+}+T G S_{2} & -T G \\
S_{1} & 0_{(p-m) \times m} \\
0_{n_{1} \times m} & I_{m}
\end{array}\right]\right)=\operatorname{rank}\left(\left[\begin{array}{cc}
z I_{n_{1}}-T A T^{+}+T G S_{2} & 0_{n_{1} \times m} \\
S_{1} & 0_{(p-m) \times m} \\
0_{n_{1} \times m} & I_{m}
\end{array}\right]\right) \\
& =\operatorname{rank}\left(\left[\begin{array}{c}
z I_{n_{1}}-T A T^{+}+T G S_{2} \\
S_{1}
\end{array}\right]\right)+m \text {. }
\end{aligned}
$$

Therefore, Eqs. (7) and (8) guarantee there exists a gain matrix $K_{k+1}$ such that: (a) $K_{k+1} C G=T G$; and (b) $E_{k}=T A T^{+}-K_{k+1} C A T^{+}$is stable.

\section{Condition relaxation}

It is of particular interest to compare the proposed RODO with the conventional FODOs. Apart from the fact that the proposed RODO is a lower-order filter, the existence condition of the RODO can be more easily satisfied than that of the FODOs, as shown in Theorem 2.

Theorem 2. If the existence condition of FODOs holds for a system given by (1), then the existence condition (8) of RODOs is also satisfied.

Proof: First, we note that the following identity holds for any non-singular matrix $P_{T}$ :

$$
\left[\begin{array}{cc}
P_{T} & 0 \\
0 & I_{p}
\end{array}\right]\left[\begin{array}{cc}
I_{n} & 0 \\
-C & z I
\end{array}\right] \underbrace{\left[\begin{array}{cc}
z I_{n}-A & -G \\
C & 0
\end{array}\right]}_{P_{f}}\left[\begin{array}{cc}
P_{T}^{-1} & 0 \\
0 & I_{p}
\end{array}\right]=\underbrace{\left[\begin{array}{cc}
z I_{n}-P_{T} A P_{T}^{-1} & -P_{T} G \\
C A P_{T}^{-1} & C G
\end{array}\right]}_{P_{F}} .
$$


Using the under-brace notations, (12) indicates that $\operatorname{rank}\left(P_{F}\right)=\operatorname{rank}\left(P_{f}\right)$, where $P_{f}$ has a full columnrank for $|z| \geq 1$ is part of the existence condition of FODO in Darouach and Zasadzinski (1997); Su et al (2015a).

Now we choose $P_{T}=\left[T^{T}, L^{T}\right]^{T}$ and let $P_{T}^{+}=\left[T^{+} L^{+}\right]$denote the Moore-Penrose Pseudo inverse of matrix $P_{T}$. Substituting $P_{T}$ and $P_{T}^{+}$into $P_{F}$ gives

$$
P_{F}=\left[\begin{array}{ccc}
z I_{n-n_{1}}-T A T^{+} & -T A L^{+} & -T G \\
-L A T^{+} & z I_{n_{1}}-L A L^{+} & -L G \\
C A T^{+} & C A L^{+} & C G
\end{array}\right]=\left[\begin{array}{ccc}
z I_{n-n_{1}}-T A T^{+} & 0 & -T G \\
-L A T^{+} & z I_{n_{1}}-L A L^{+} & -L G \\
C A T^{+} & 0 & C G
\end{array}\right]
$$

From (8) and (13), it can be seen that matrix $P$ in (8) is a sub-matrix of $P_{F}$. This indicates that matrix $P$ is of full column-rank if $P_{F}$ has a full column-rank.

\section{Disturbance observer design}

In this subsection, the two gain matrices of the RODO in (4) will be investigated using the MVUE method. A Lemma on the inverse of portioned matrix will be first introduced.

Lemma 1. (Simon (2006)) Suppose matrix A is non-singular and B has a full column-rank. Then the following identity holds:

$$
\left[\begin{array}{cc}
A & B \\
B^{T} & 0
\end{array}\right]^{-1}=\left[\begin{array}{cc}
A^{-1}-A^{-1} B\left(B^{T} A^{-1} B\right)^{-1} B^{T} A^{-1} & A^{-1} B\left(B^{T} A^{-1} B\right)^{-1} \\
\left(B^{T} A^{-1} B\right)^{-1} B^{T} A^{-1} & -\left(B^{T} A^{-1} B\right)^{-1}
\end{array}\right]
$$

Under the existence condition given in Section. Existence Condition, one can obtain the dynamics of $e_{k+1}^{x}=T x_{k+1}-\hat{z}_{k+1}$ from (5) and (6):

$$
e_{k+1}^{x}=E_{k} e_{k}^{x}+\left(T-K_{k+1} C\right) \omega_{k}-K_{k+1} v_{k+1} .
$$

In addition, the dynamics $e_{k}^{d}=d_{k}-\hat{d}_{k}$ are governed by

$$
e_{k}^{d}=-J_{k+1} N e_{k}^{x}-J_{k+1}\left(C \omega_{k}+v_{k+1}\right) .
$$




\section{State function observer :}

The estimation error covariance matrix $P_{k \mid k}^{x}=E\left(e_{k}^{x} e_{k}^{x T}\right)$ can be calculated from (14):

$$
\begin{aligned}
P_{k+1 \mid k+1}^{x}= & E_{k} P_{k \mid k}^{x} E_{k}^{T}+\left(T-K_{k+1} C\right) Q_{k}\left(T-K_{k+1} C\right)^{T}+K_{k+1} R_{k+1} K_{k+1}^{T} \\
= & \left(T A T^{+}-K_{k+1} C A T^{+}\right) P_{k \mid k}^{x}\left(T A T^{+}-K_{k+1} C A T^{+}\right)^{T} \\
& +T Q_{k} T^{T}-T Q_{k} C^{T} K_{k+1}^{T}-K_{k+1} C Q_{k} T^{T} \\
& +K_{k+1} C Q_{k} C^{T} K_{k+1}^{T}+K_{k+1} R_{k+1} K_{k+1}^{T} .
\end{aligned}
$$

Let $\bar{A}=T A T^{+}, \quad \bar{C}=C A T^{+}, \Phi=\bar{C} P_{k \mid k}^{x} \bar{C}^{T}+C Q_{k} C^{T}+R_{k+1}, \Psi=C Q_{k} T^{T}+\bar{C} P_{k \mid k}^{x} \bar{A}^{T}$ and $P^{*}=\bar{A} P_{k \mid k}^{x} \bar{A}^{T}+T Q_{k} T^{T}$. Eq. (16) can be simplified to be:

$$
P_{k+1 \mid k+1}^{x}=K_{k+1} \Phi K_{k+1}^{T}-K_{k+1} \Psi-\Psi^{T} K_{k+1}^{T}+P^{*}
$$

In addition, the unbiasedness condition of state estimation imposes a constraint on the gain matrix $K_{k+1}$ (see Crassidis and Junkins (2011)), i.e.

$$
K_{k+1} C G=T G
$$

We solve the MVUE problem by finding $K_{k+1}$ which minimizes the trace of (17), subject to the constraint (18) based on the Lagrange multiplier approach in Kitanidis (1987) and Crassidis and Junkins (2011) (see, pp. 68). The Lagrangian for the constraint optimization problem is

$$
\operatorname{Tr}\left[K_{k+1} \Phi K_{k+1}^{T}-2 \Psi^{T} K_{k+1}^{T}+P^{*}\right]-2 \operatorname{Tr}\left[\left(K_{k+1} C G-T G\right) \Lambda_{k+1}^{T}\right]
$$

where $\Lambda_{k+1}$ is the Lagrange multiplier. Setting the derivative of (19) with respect to $K_{k+1}$ equal to zero yields:

$$
2 \Phi K_{k+1}^{T}-2 \Psi-2 C G \Lambda_{k+1}^{T}=0 .
$$

Combining (18) and (20), we can obtain the following equation:

$$
\left[\begin{array}{cc}
\Phi & -C G \\
G^{T} C^{T} & 0
\end{array}\right]\left[\begin{array}{c}
K_{k+1}^{T} \\
\Lambda_{k+1}^{T}
\end{array}\right]=\left[\begin{array}{c}
\Psi \\
G^{T} T^{T}
\end{array}\right]
$$

Following Kitanidis (1987) in conjunction with Lemma 1, we can obtain $K_{k+1}$ as follows:

$$
K_{k+1}=\Psi^{T} \Phi^{-1}+\left(T G-\Psi^{T} \Phi^{-1} C G\right)\left(G^{T} C^{T} \Phi^{-1} C G\right)^{-1} G^{T} C^{T} \Phi^{-1} .
$$


Inserting $K_{k+1}$ in (22) into (17), one can obtain:

$$
P_{k+1 \mid k+1}^{x}=P^{*}-\Psi^{T} \Phi^{-1} \Psi+\left(T G-\Psi^{T} \Phi^{-1} C G\right)\left(G^{T} C^{T} \Phi^{-1} C G\right)^{-1}\left(T G-\Psi^{T} \Phi^{-1} C G\right)^{T} .
$$

\section{Disturbance observer :}

We now work out $J_{k+1}$ in (4) in a similar manner. First, from (15) we can obtain the disturbance estimation error covariance matrix $P_{k \mid k}^{d}=E\left(e_{k}^{d} e_{k}^{d T}\right)$ :

$$
P_{k \mid k}^{d}=J_{k+1} N P_{k \mid k}^{x} N^{T} J_{k+1}^{T}+J_{k+1}\left(C Q_{k} C^{T}+R_{k+1}\right) J_{k+1}^{T} .
$$

Noting $N=C A T^{+}$and by the definition of $\Phi$, Eq. (24) can be re-arranged as

$$
P_{k \mid k}^{d}=J_{k+1} \Phi J_{k+1}^{T}
$$

In addition, the unbiased estimation of $d_{k}$ also imposes a constraint on gain matrix $J_{k+1}$, i.e. $J_{k+1} C G=$ $I$. We can obtain the optimal $J_{k+1}$ below via minimizing the trace of (25), subject to this constraint:

$$
J_{k+1}=\left(G^{T} C^{T} \Phi^{-1} C G\right)^{-1} G^{T} C^{T} \Phi^{-1}
$$

Inserting (26) into (25), one can obtain an explicit expression of the disturbance estimation error covariance matrix:

$$
P_{k \mid k}^{d}=\left(G^{T} C^{T} \Phi^{-1} C G\right)^{-1} .
$$

The obtained RODO is summarized in Theorem 3, where the poof has been presented throughout the aforementioned deviation.

Theorem 3. Under the existence condition given in Theorem 1, there exists a minimum-variance unbiased estimator of the disturbances $d_{k}$ given by

$$
\left\{\begin{array}{l}
\hat{z}_{k+1}=T A T^{+} \hat{z}_{k}+K_{k+1}\left(y_{k+1}-C A T^{+} \hat{x}_{k}\right) \\
\hat{d}_{k}=J_{k+1}\left(y_{k+1}-C A T^{+} \hat{z}_{k}\right)
\end{array}\right.
$$

where the gain matrices $K_{k+1}$ and $J_{k+1}$ are given by (22) and (26) respectively, and the corresponding state function estimation error covariance matrix and disturbance estimation error covariance matrix are given by (23) and (27) respectively. 


\section{Relationships with the existing results}

Next, we investigate the relationships between the proposed RODO and the existing approaches. When the states are fully estimable in the presence of disturbances, the state and disturbance filtering problem has been investigated by a number of researchers (e.g. Gillijns and De Moor (2007); Li (2013); Su et al (2015a); Kitanidis (1987)). The relationships between existing FODOs and proposed RODO are summarized in Theorem 4.

Theorem 4. When the states are fully estimable in the presence of disturbances, the proposed RODO is equivalent to the FODO in Kitanidis (1987) for sole state estimation, and to the one in Gillijns and De Moor (2007) for the estimation of both states and disturbances.

Proof: When the states are fully estimable, $T$ can be chosen as the identity matrix and hence the RODO reduces to:

$$
\left\{\begin{array}{l}
\hat{x}_{k+1}=A \hat{x}_{k}+K_{k+1}\left(y_{k+1}-C A \hat{x}_{k}\right) \\
\hat{d}_{k}=J_{k+1}\left(y_{k+1}-C A \hat{x}_{k}\right)
\end{array},\right.
$$

where $J_{k+1}=\left(P_{k \mid k}^{d}\right)^{-1} G^{T} C^{T} H_{k+1}^{-1}$, and

$$
K_{k+1}=P_{k+1 \mid k} C^{T} H_{k+1}^{-1}+\left(G-P_{k+1 \mid k} C^{T} H_{k+1}^{-1} C G\right)\left(G^{T} C^{T} H_{k+1}^{-1} C G\right)^{-1} C G H_{k+1}^{-1}
$$

with $P_{k+1 \mid k}=A P_{k \mid k} A^{T}+Q_{k}, H_{k+1}=C P_{k+1 \mid k} C^{T}+R_{k+1}$.

In addition, Eq. (23) reduces to

$$
P_{k+1 \mid k+1}^{x}=P_{k+1 \mid k}^{x}-P_{k+1 \mid k}^{x} C^{T} H_{k+1}^{-1} C P_{k+1 \mid k}^{x}+B\left(G^{T} C^{T} \tilde{R}_{k}^{-1} C G\right)^{-1} B^{T},
$$

with $B=G-P_{k+1 \mid k}^{x} C^{T} H_{k+1}^{-1} C G$ and Eq. (27) reduces to $P_{k \mid k}^{d}=\left(G^{T} C^{T} H_{k}^{-1} C G\right)^{-1}$. These recursive formulae are identical to the results in Kitanidis (1987) for sole state estimation, and the same as the results in Gillijns and De Moor (2007) for the estimation of states and disturbances.

Next, we briefly compare the proposed RODO with the recently developed RODO for deterministic discrete-time systems in Kim and Rew (2013). We first point out that the existence condition in Kim and Rew (2013) requires the existence of a gain matrix such that the corresponding composite matrix is asymptotically stable. This gain matrix also involves a static output feedback problem, for which the general solvability is not known yet (see Kim and Rew (2013) for details). In contrast, the existence condition proposed in this letter is easy to check and it collapses to that of the conventional FODOs for fully estimable states. In addition, unlike the RODO in Kim and Rew (2013) that assumes the disturbances are slowly time-varying, no particular assumption on the disturbance dynamics is imposed 
in the proposed method, hence extending its applicability. The simulation comparisons for the proposed algorithm with that of Kim and Rew (2013) are given in the next Section.

\section{Numerical verification}

In this section, a numerical example is first given to compare the disturbance estimation performance of the proposed algorithm with the algorithm proposed in Kim and Rew (2013), which will demonstrate the advantages of the proposed algorithm in generic disturbance estimation. Then the proposed algorithm is applied to the disturbance estimation problem for a double-effect pilot plant evaporator system with unobservable states.

\section{Simulation study 1: performance comparison}

We first of all use a simple numerical example to compare the proposed algorithm with the RODO in Kim and Rew (2013). Consider system (1) with $A=\left[\begin{array}{ccc}1.1 & 0.5 & 0 \\ 0 & 0.9 & 0 \\ 0 & 0.5 & 2\end{array}\right], G=\left[\begin{array}{l}1 \\ 0 \\ 1\end{array}\right]$, $C=\left[\begin{array}{lll}1 & 0 & 0 \\ 0 & 1 & 0\end{array}\right], Q_{k}=0.02 \times I_{3}$, and $R_{k}=0.01 \times I_{2}$. The disturbance profile in simulation study as shown in the upper plot of Fig. 1 was used to represent a generic disturbance which included a slow time-varying disturbance (i.e., step-type disturbance) and a fast time-varying disturbance (i.e., sinusoidal disturbance). The step amplitudes at 0 and 70th step were taken as 7 and -7 respectively, whereas the sinusoidal function between 30th step and 70th step was chosen as $4 \sin (40 \pi t / 180)+2$ with $t$ being each step index. The disturbance profile was designed to verify the effectiveness of different disturbance observer algorithms and therefore was assumed to be completely unknown to the observer design.

It can be easily verified that this system does not satisfy the existence condition of the FODO in Gillijns and De Moor (2007) and Darouach (2000), and hence no FODO exists.

In the proposed RODO, we chose $T=\left[\begin{array}{lll}1 & 0 & 0 \\ 0 & 1 & 0\end{array}\right]$ which satisfies the existence condition in Theorem 1. The initial states of the system and observer are taken as $x_{0}=[1,2,1]^{T}$ and $z_{0}=[0,0]^{T}$ respectively. The RODO in Kim and Rew (2013) for discrete-time system with slowly time-varying disturbance assumption is also applied for the purpose of comparison, where the matrix $K$ therein is chosen as $K=[0.9,0,0]$. The comparison results are shown in Fig. 1, where upper figure depicts the disturbance estimates and the lower figure displays the disturbance estimation errors.

We can see from Fig. 1 that in the presence of unknown disturbance consisting of step-type and sinusoidal-type, the proposed RODO can obtain unbiased estimation. For the algorithm in Kim and Rew 

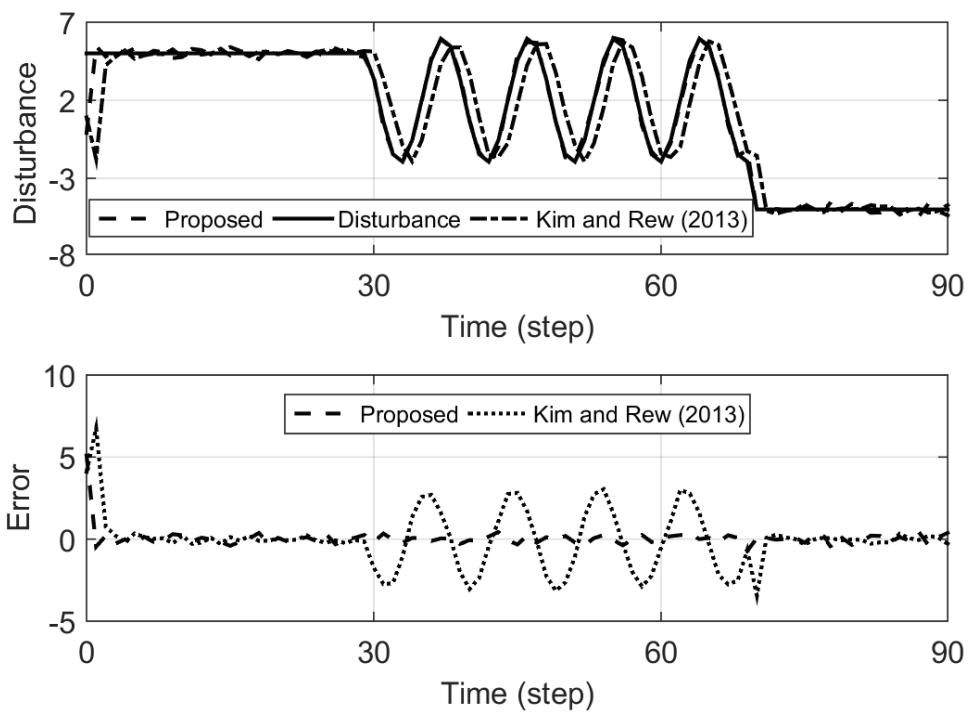

Figure 1. The disturbance estimation based on the proposed RODO and the algorithm in Kim and Rew (2013): real line (actual disturbance), dashed line (the proposed RODO) and dotted line (the result in Kim and Rew (2013)). A zoom-in plot during steps 10 and 20 is also given in the upper plot.

(2013), on the other hand, it can be seen that it works well and obtains unbiased estimate for constant disturbance. However, the disturbance estimation error is quite large in the presence of sinusoidal-type disturbance; this is due to the fact that the RODO proposed in Kim and Rew (2013) requires that the disturbance is slowly time-varying and will result in disturbance estimation error for fast time-varying disturbances. This example demonstrates the advantages of the proposed RODO in generic disturbance estimation that includes both slow and fast time-varying disturbances over the traditional algorithms.

In some applications in practice, the covariance matrices of input noises and measurement noises may not be exactly unknown. It is therefore of practical interest to investigate the performance of the proposed algorithm in such scenarios. For this end, we tested the robustness of the proposed RODO by choosing different covariance matrices for data generation. Specifically, we first considered the effect of input noises. In this scenario, the measurement covariance matrix was fixed as $R_{k}=0.01 \times I_{2}$ but a different input noise covariance matrix was used, i.e. $Q_{k}=0.04 \times I_{3}$ and $Q_{k}=0.06 \times I_{3}$ respectively. Next, we considered the effect of measurement noises. In this scenario, the input covariance matrix was fixed as $Q_{k}=0.02 \times I_{3}$ but a different measurement noise covariance matrix was used, i.e. $R_{k}=0.03 \times I_{2}$ and $R_{k}=0.05 \times I_{2}$ respectively.

During the estimation stage, however, we supposed that the true covariance matrices used to simulate the system states and measurements were not perfectly known; rather, it was the covariance matrices 
$Q_{k}=0.02 \times I_{3}$, and $R_{k}=0.01 \times I_{2}$ that were used to estimate the states and the unknown disturbances. The mean squared error (MSE) was used as the criterion in the performance comparison between the proposed RODO with the one in Kim and Rew (2013).

Simulations were run for 50 times for each scenario and the average MSEs were calculated and summarized in Table 1.

Table 1. Comparison of the average MSE in the robustness test under different noise covariance matrices.

\begin{tabular}{|c|c|c|c|c|}
\hline Method/Noise & $Q_{k}=0.04 \times I_{3}$ & $Q_{k}=0.06 \times I_{3}$ & $R_{k}=0.03 \times I_{2}$ & $R_{k}=0.05 \times I_{2}$ \\
\hline Proposed & 0.3475 & 0.3637 & 0.3668 & 0.4135 \\
\hline Kim and Rew (2013) & 2.7946 & 2.8351 & 2.8301 & 2.8461 \\
\hline
\end{tabular}

One can see from Table 1 that both methods still worked well in terms of convergences when the true covariance matrices were not perfectly known in the state and disturbance estimation. However, the performances for both methods became worse when the covariance matrices used in the estimation deviated more substantially from the true covariance matrices. Overall, the proposed RODO still outperformed the one in Kim and Rew (2013).

\section{Simulation study 2: double-effect pilot plant evaporator}

Next, we apply the proposed RODO to the disturbance estimation problem for a double-effect pilot plant evaporator investigated in Xiong and Saif (2003) and Phatak and Viswanadham (1988). The problem is briefly outlined as follows. The feed solution (flow $F_{0}$ and concentration $C_{0}$ ) is pumped into the first effect, where the first effect solution (hold-up $W_{1}$ ) is heated by saturated steam (temperature $\left.T_{s}\right)$ and the boil-off travels into the second effect steam jacket. The concentrated solution from the first effect (flow $F_{1}$ and concentration $C_{1}$ ) enters the second effect which operates under vacuum. The hold-up in the second effect is $W_{2}$. The concentrated product (flow $F_{2}$ and concentration $C_{2}$ ) is pumped to storage. Based on the physical properties, the evaporator can be modelled by a fifth-order linear state-space model with unobservable states, where the system variables and disturbance variables are $x=\left[W_{1}, C_{1}, T_{1}, W_{2}, C_{2}\right]$ ( $T_{1}$ denotes the temperature of the first effect solution) and $d=\left[C_{0}, F_{0}\right]$ respectively. A schematic diagram of the pilot plant double effect evaporator system is available in Buchholt and Kmmel (1981), and Phatak and Viswanadham (1988).

In our case study, we chose the system matrix in continuous time domain used in Xiong and Saif (2003), and then we discretized the continuous-time model with a sampling time of $30 \mathrm{~s}$ (see, Phatak and Viswanadham (1988)). This resulted in the following discrete-time system 


$$
A=\left[\begin{array}{ccccc}
1 & 0 & -0.0030 & 0 & 0 \\
0 & 0.2923 & 0.0003 & 0 & 0 \\
0 & 0 & 0 & 0 & 0 \\
0 & 0 & -0.0031 & 1 & 0 \\
0 & 0.7121 & 0.0019 & 0 & 0.2165
\end{array}\right], G=\left[\begin{array}{cc}
0 & 30.6207 \\
1.0702 & -2.4170 \\
0 & -6.2671 \\
0 & 0.6572 \\
1.1068 & -3.0385
\end{array}\right], C=\left[\begin{array}{ccccc}
1 & 0 & 0 & 0 & 0 \\
0 & 1 & 0 & 0 & 0
\end{array}\right] .
$$

In the simulation, we chose $Q_{k}=0.1 \times I_{5}$ and $R_{k}=0.05 \times I_{2}$, and set $d_{k, 2}=-0.5 \times d_{k, 1}$, where the disturbance $d_{k, 1}$ profile is depicted in the upper left plot of Fig. 2. The initial values of system (1) and observer (4) were taken as $x_{0}=[1,2,5,1,1]^{T}$ and $z_{0}=[0,0,0]^{T}$ respectively. The initial covariance matrix is chosen as $P_{0 \mid 0}^{x}=I_{3}$.

It can be easily verified that this system does not satisfy the existence condition of the FODOs in Gillijns and De Moor (2007); Darouach and Zasadzinski (1997) and Su et al (2015a). Hence the system state variables $x_{k, i}(i=1, \ldots, 5)$ are not fully estimable and no FODOs exist.

Now we apply the proposed RODO. We chose $T=\left[I_{3}, O_{3 \times 2}\right]$ (i.e., only the first three states were estimated) which satisfies the existence condition in Theorem 1. The simulation results for disturbance $d_{k, 1}$ and state $x_{k, 3}$ are shown in Fig. 2; similar results were obtained for disturbance $d_{k, 2}$ and states $x_{k, 1}$ and $x_{k, 2}$ (not shown here). The left (or right) two graphs display the estimated disturbance (or state) and the corresponding estimation error where the simulated (estimated) values are plotted using a real (dotted) line.
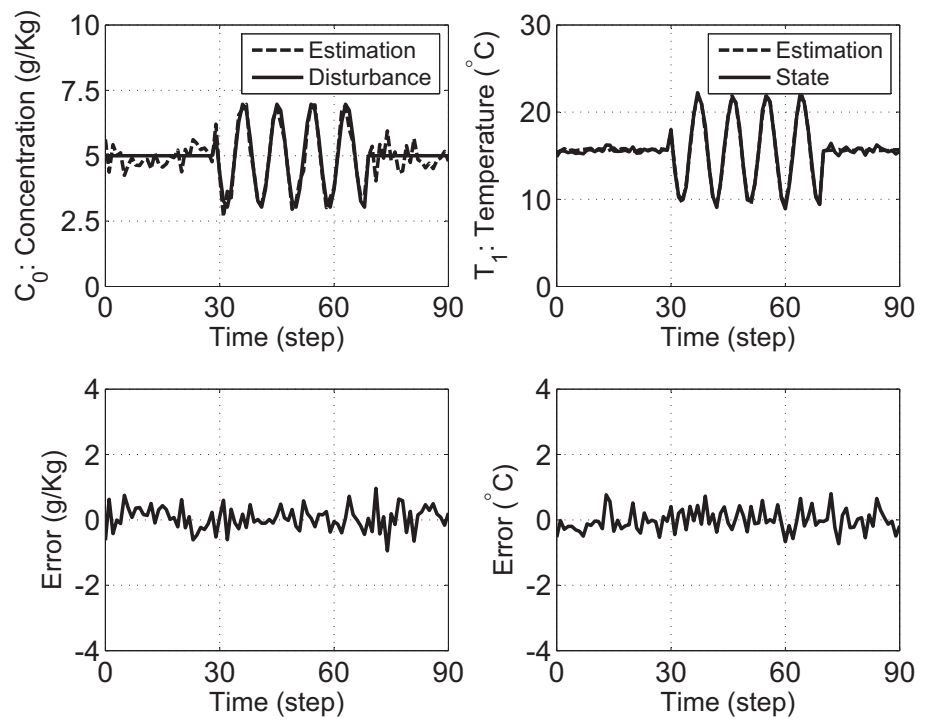

Figure 2. The disturbance and state estimate based on the proposed RODO: left two graphs (concentration $d_{k, 1}$ estimate and estimation error); right two graphs (temperature $T_{1}$ estimate and estimation error). 
We can see from Fig. 2 that the proposed RODO can obtain a reasonably good (by taking the large input and measurement noises into account) unbiased disturbance estimate even if no FODOs exist.

\section{Conclusions and discussion}

In this section, we highlight the major contributions of the proposed reduced-order disturbance observer (RODO) and consider some future research in this area.

First, unlike the conventional disturbance observers for discrete-time linear stochastic systems that require the states are fully estimable, this paper has proposed a reduced-order disturbance observer where the states are not fully estimable. Hence, this research extends the applicability of the disturbance observer techniques to a wider application area. In particular, the proposed RODO can be applied to fault diagnosis problem by treating RODO as fault diagnosis observer.

In addition, we have established an existence condition of a general form of RODO. On the basis of this, we have explored the relationships between the existing FODOs in the literature and the proposed RODO in this paper: it is shown that the formers are a special case of the proposed RODO when the states are fully estimable. In comparison with the recently developed RODO in Kim and Rew (2013), the proposed RODO does not impose any particular assumption on the disturbance dynamics.

Finally, we note that, since no disturbance information is assumed to be available in the proposed RODO, its existence condition is more restrict (e.g., the dimension of the measurement vector is larger than that of disturbance vector) in comparison with the one with partial disturbance information (e.g., Su et al (2015a)). Future work can be done to relax the existence condition by incorporating some suitable available disturbance information.

\section{Acknowledgements}

This work was partly supported by the UK Engineering and Physical Sciences Research Council (EPSRC) Autonomous and Intelligent Systems programme under the grant number EP/J011525/1 with BAE Systems as the leading industrial partner. The fourth author was supported in part by National Natural Science Foundation of China under Grant 61573099, Natural Science Foundation of Jiangsu Province under Grants BK2012327, and Fundamental Research Funds for the Central Universities.

\section{References}

Anderson, B. D., \& Moore, J. B. (2012). Optimal filtering. Courier Corporation.

Bejarano, F. J., Fridman, L., \& Poznyak, A. (2009). Unknown input and state estimation for unobservable systems. SIAM Journal on Control and Optimization, 48(2), 1155-1178. 
Buchholt, F., \& Kmmel, M. (1981). A multivariable selftuning regulator to control a double effect evaporator. Automatica, 17(5), 737-743.

Chang, J. L. (2006). Applying discrete-time proportional integral observers for state and disturbance estimations. IEEE Transactions on Automatic Control, 51(5), 814-818.

Chen, W. H., Ballance, D. J., Gawthrop, P. J., \& O’Reilly, J. (2000). A nonlinear disturbance observer for robotic manipulators. IEEE Transactions on Industrial Electronics, 47(4), 932-938.

Chen, W. H., Yang, J., Guo, L., \& Li, S. (2016). Disturbance observer-based control and related methods: An overview. IEEE Transactions on Industrial Electronics, 63(2), 1083-1095.

Crassidis, J. L., \& Junkins, J. L. (2011). Optimal estimation of dynamic systems. CRC press.

Dan Simon, (2006). Optimal state estimation: Kalman, H infinity, and nonlinear approaches. John Wiley \& Sons.

Darouach, M., \& Zasadzinski, M. (1997). Unbiased minimum variance estimation for systems with unknown exogenous inputs. Automatica, 33(4), 717-719.

Darouach, M. (2000). Existence and design of functional observers for linear systems. IEEE Transactions on Automatic Control, 45(5), 940-943.

Fang, H., De Callafon, R. A., \& Corts, J. (2013). Simultaneous input and state estimation for nonlinear systems with applications to flow field estimation. Automatica, 49(9), 2805-2812.

Gillijns, S., \& De Moor, B. (2007). Unbiased minimum-variance input and state estimation for linear discrete-time systems. Automatica, 43(1), 111-116.

Guo, L., \& Cao, S. (2013). Anti-disturbance control for systems with multiple disturbances. CRC Press.

Jiang, B., \& Chowdhury, F. N. (2005). Fault estimation and accommodation for linear MIMO discrete-time systems. IEEE Transactions on Control Systems Technology, 13(3), 493-499.

Kim, K. S., \& Rew, K. H. (2013). Reduced order disturbance observer for discrete-time linear systems. Automatica, 49(4), 968-975.

Kim, K. S., Rew, K. H., \& Kim, S. (2010). Disturbance observer for estimating higher order disturbances in time series expansion. IEEE Transactions on Automatic Control, 55(8), 1905-1911.

Kimura, H. (1978). Deadbeat function observers for discrete-time linear systems. SIAM Journal on Control and Optimization, 16(6), 880-894.

Kitanidis, P. K. (1987). Unbiased minimum-variance linear state estimation. Automatica, 23(6), 775-778.

Li, B. (2013). State estimation with partially observed inputs: A unified Kalman filtering approach. Automatica, 49(3), 816-820.

Li, S., Yang, J., Chen, W. H., \& Chen, X. (2014). Disturbance observer-based control: methods and applications. CRC press. 
Ohishi, K., Nakao, M., Ohnishi, K., \& Miyachi, K. (1987). Microprocessor-controlled DC motor for load-insensitive position servo system. IEEE Transactions on Industrial Electronics, 1(IE-34), 44-49.

Phatak, M. S., \& Viswanadham, N. (1988). Actuator fault detection and isolation in linear systems. International journal of system science 19(12) 2593-2603.

Su, J., Yang, J., \& Li, S. (2013). Continuous finite-time anti-disturbance control for a class of uncertain nonlinear system. Transactions of the Institute of Measurement and Control, 36(3), 300-311.

Su, J., Li, B., \& Chen, W. H. (2015a). On existence, optimality and asymptotic stability of the Kalman filter with partially observed inputs. Automatica, 53, 149-154.

Su, J., Li, B., \& Chen, W. H. (2015b). Simultaneous state and input estimation with partial information on the inputs. Systems Science \& Control Engineering, 3(1), 445-452.

$\mathrm{Su}$, J., \& Chen, W. H. (2016). Fault diagnosis for vehicle lateral dynamics with robust threshold. IEEE International Conference on Industrial Technology, accepted, March 14-17, Taipei.

Xiong, Y., \& Saif, M. (2003). Unknown disturbance inputs estimation based on a state functional observer design. Automatica, 39(8), 1389-1398.

Yang, J., Li, S., Su, J., \& Yu, X. (2013). Continuous nonsingular terminal sliding mode control for systems with mismatched disturbances. Automatica, 49(7), 2287-2291.

Yang, J., Su, J., Li, S., \& Yu, X. (2014). High-order mismatched disturbance compensation for motion control systems via a continuous dynamic sliding-mode approach. IEEE Transactions on Industrial Informatics, 10(1), 604-614. 